\title{
Spinal tuberculosis: imaging features on MRI
}

\author{
Bhatnagar S. ${ }^{1}$, Garg A. ${ }^{2}$, Kaur A. ${ }^{3}$, Kaur N. ${ }^{4}$, Mohi J. ${ }^{5}$ \\ ${ }^{1}$ Dr. Simmi Bhatnagar, Assistant Professor, ${ }^{2}$ Dr. Ambry Garg, Junior Resident, ${ }^{3}$ Dr. Amarjit Kaur, Professor, \\ ${ }^{4}$ Dr. Navkiran Kaur, Professor and HOD, ${ }^{5}$ Dr. Jaswinder Mohi, Associate Professor; all authors are affiliated with \\ Department of Radiodiagnosis, GMC Patiala, Punjab, India.
}

Corresponding Author: Dr. Ambry Garg, Junior Resident, Department of Radiodiagnosis, GMC Patiala, Punjab, India, Email: ambrygarg@gmail.com

\begin{abstract}
Introduction: Spine is the most common site of skeletal tuberculosis.Radiographic manifestations of tuberculous spondylitis include intraosseous and paraspinal abscess formation, subligamentous spread of infection, vertebral body destruction and collapse, and extension into the spinal epidural space. Significant instability and deformity of the spine can result, mandating prompt diagnosis and treatment to prevent permanent neurologic damage. Objective: This is a prospective and descriptive study to evaluate the role of MRI in spinal tuberculosis and various spectrum ofits manifestations. Materials and Methods: This prospective study was on 25 patients of either age or sex with clinical suspicion of spinal TB over a period of 2 years and subsequently went MR Imaging and features were correlated with other investigations and histopathological diagnosis in few patients. Results: Thoracic spine was the most common site of involvement. Vertebral body wedge collapse or compression fracture was seen in $64 \%$ of patients. Posterior element involvement was found in 10 out of 25 cases. Prevertebral \& paravertebral collections were seen in $88 \%$ of cases. Epidural soft tissue component was seen in $56 \%$ of cases. Conclusion: MRI is the best diagnostic modality for spinal TB and to demonstratethe various associated manifestations which help in assessing the extent and severity of the disease and thus inearly and correct management planning.
\end{abstract}

Keywords: Epidural, Pre/paravertebral, Subligamentous and vertebral compression/ collapse

\section{Introduction}

Spinal TB (Pott's disease) is the most common as well as one of the most dangerous forms of skeletal TB and accounts for $50 \%$ of all the cases of skeletal TB. Although the thoracolumbar junction seems to be the most common site of the spinal column involvement in spinal TB, any part of the spine can be affected. Furthermore, the incidence of neurologic complications in spinal TB varies from $10 \%$ to $43 \%$ [1].

Spinal tuberculosis is usually a secondary infection from a primary site in the lung or genitourinary system. Spread to the spine is thought to be hematogenous in most instances. Tissue necrosis and breakdown of inflammatory cells result in a paraspinal abscess. The pus may be localized or it may track along tissue planes. Progressive necrosis of bone leads to a kyphotic deformity. Typically, the infection begins in the anterior aspect of the vertebral body adjacent to the disc. The

Manuscript received: $20^{\text {th }}$ January 2018

Reviewed: $30^{\text {th }}$ January 2018

Author Corrected: $6^{\text {th }}$ February 2018

Accepted for Publication $11^{\text {th }}$ February 2018 infection then spreads to the adjacent vertebral bodies under the longitudinal ligaments. Noncontiguous (skip) lesions are also occasionally seen [2]. Lower thoracic and lumbar vertebrae are the most common sites of spinal TB followed by middle thoracic and cervical vertebrae. There are mainly four sites of infections in vertebra-paradiscal, central, anterior subligamentous and appendiceal [3].

CT demonstrates abnormalities earlier than plain radiography. The pattern of bone destruction may be fragmentary in $47 \%$ of the cases; osteolytic in $34 \%$, localized and sclerotic in $10 \%$ and subperiosteal in $30 \%$ cases. Other findings include soft tissue involvement and paraspinal tissue abscess. CT is of great value in the demonstration of any calcification within the cold abscess or visualizing epidural lesions containing bone fragments [4]. However, CT is less accurate in defining the epidural extension of the disease and its effect on neural structures. In contrast to most imaging methods, MRI has the advantages of improved contrast resolution 
Original Research Article

for bone and soft tissues along with versatility of direct imaging in multiple planes. With the aid of intravenous administration of magnetic resonance contrast agents, MRI is highly accurate in distinguishing granulation tissue from cold abscess. MRI can reveal more extensive involvement than the plain films. MRI clearly demonstrated the extent of soft tissue disease and its effect on the thecal sac, cord, and foramina in cases with doubtful CT findings [5].

\section{MRI characteristics in spinal TB are as follows}

- Vertebral body endplate involvement appears as heterogeneously enhancing endplate irregularity on post-contrast sequences.

- Vertebral lesions appear hypo intense on T1W images, hyperintense on $\mathrm{T} 2 \mathrm{~W}$ images and shows heterogeneous enhancement on postcontrast $\mathrm{T} 1 \mathrm{~W}$ images.

- Marrow edema appears as hyperintense areas on T2W and STIR images.

- Intervertebral disc involvement appears hypointense on T1W and hyperintense on T2W images and shows heterogeneous enhancement on post-contrast T1W images.

- Prevertebral, paravertebral and psoas abscesses appear as heterogeneous lesions with peripheral enhancement and central non-enhancing hypointense areas on post-contrast T1W images. The level, extent and size of abscess can be well delineated on MRI.

Granulation tissue appears heterogeneously enhancing soft tissue on post-contrast T1W images. The granulation tissues and epidural abscess can cause narrowing of thecal sac or compression of spinal cord causing neurological complications[3].

\section{Materials and Methods}

Place of study: Department of Radiodiagnosis, Rajindera Hospital, Patiala

Type of study: Prospective study

Sampling method: Patients referred for MRI scan with clinical suspicion of spinal TB

\section{Inclusion criteria}

1) Patients with or without neurological deficit at spinal level with strong clinical suspicion of spinal tb.

2) All age groups.

3) Both sexes.

\section{Exclusion criteria}

1) Trauma patients

2) Patients with metallic implants

Technique- The MRI scan was performed on $1.5 \mathrm{~T}$ GE MRI scanner. The following MRI sequences were studied: Sagittal and axial T1 weighted (T1 FSE), Sagittal and axial T2 weighted (T2 FSE), Coronal and sagittal STIR sequences followed by post-contrast T1 weighted sequences in axial, coronal and sagittal planes. Postcontrast T1W sequences were obtained by using intravenous administration of Gd DTPA in a dose of 0.1 $\mathrm{mmol} / \mathrm{kg}$ body weight.

The following features were assessed by MRI:

Epidural involvement assessed for the following

1. Extent of vertebral involvement: body / posterior element involvement.

2. Wedging or compression.

3. Involvement of disc.

4. Subligamentous extension.

5. Extent of abscess: Epidural / paravertebral / psoas.

6. Spinal cord changes.

\section{Results}

Out of 25 patients of spinal TB, the age of patients varied from 12 to 70 years with the mean age of 43.36 years. Out of 25 cases, 15 were males and 10 were females with the mean age of 43.64 in males and 43 years in females.

Thoracic spine was the most common site of involvement. Thoracic spine was involved in 52\% followed by lumbar spine in $28 \%$, cervical spine in $16 \%$ and sacral vertebrae in $4 \%$ of cases among 25 patients. Among dorsal vertebrae D4-D6 level were commonly involved in upper thoracic spine while D10- D12 were more involved in lower thoracic spine. L3L5 level were commonly involved in the lumbar region. Compression /collapse of the vertebral bodies was seen in $64 \%$ of cases. Pre and paravertebralcollection was seen in $22(88 \%)$ cases. Involvement of psoas muscle leading to psoas abscess was seen in 8 among 22 patients with pre/paravertebral collection.

Epidural soft tissue component was seen in $56 \%$ of cases. The epidural soft tissue leading to spinal cord compression among the 17 cases of thoracic and cervical cord involvement was seen in 11 patients and the cord changes in the form of odema were seen in 6 cases. 
Original Research Article

Table-1: Distribution of spinal TB.

\begin{tabular}{|c|c|c|}
\hline Level of Lesion & Number of Patients & Percentage \\
\hline Cervical & 4 & 16 \\
\hline Thoracic & 13 & 52 \\
\hline Lumbar & 7 & 28 \\
\hline Sacral & 1 & 4 \\
\hline Total & $\mathbf{2 5}$ & $\mathbf{1 0 0}$ \\
\hline
\end{tabular}

Table-2: Different manifestations of spinal TB on MRI.

\begin{tabular}{|c|c|c|}
\hline MRI Finding & No. of Patients & Percentage \\
\hline Intervertebral disc involvement & 23 & 92 \\
\hline Wedge compression/ collapse of body & 16 & 64 \\
\hline Posterior element involvement & 10 & 40 \\
\hline Epidural collection & 14 & 56 \\
\hline Pre and paravertebral collection & 22 & 88 \\
\hline Spinal cord compression & 11 & 44 \\
\hline
\end{tabular}

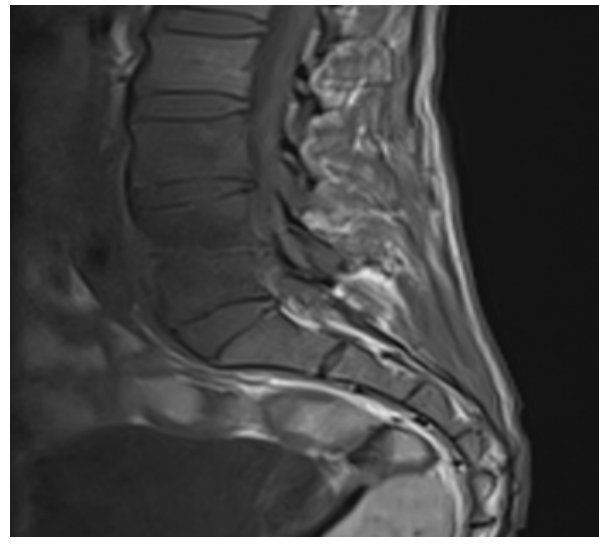

Figure-1: T1W Sag

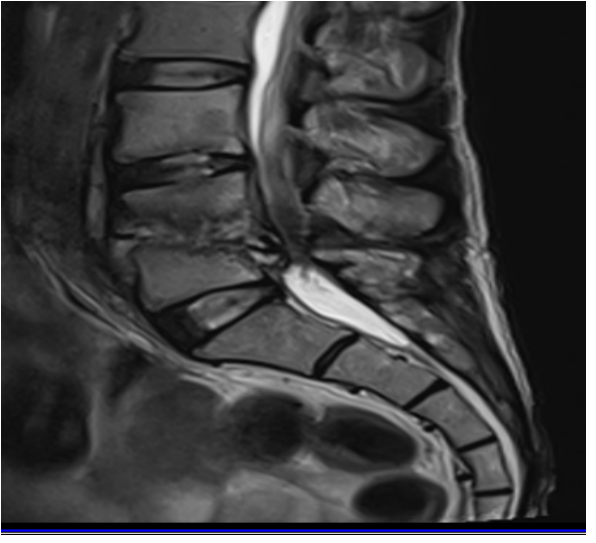

Figure-2: T2W Sag

Figure-1: Destruction of L4-L5 disc and adjacent Vertebral end plates is seen and theInvolved vertebrae showing hypointense Signal intensity.

Figure-2: Hyperintense prevertebral and epidural collection is seen

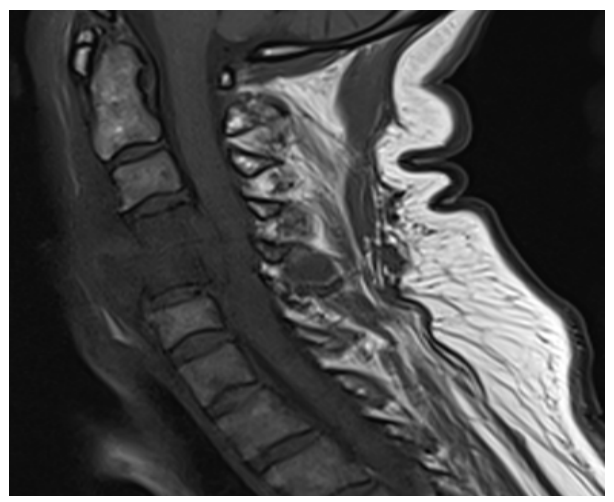

Figure-3: T1W Sag

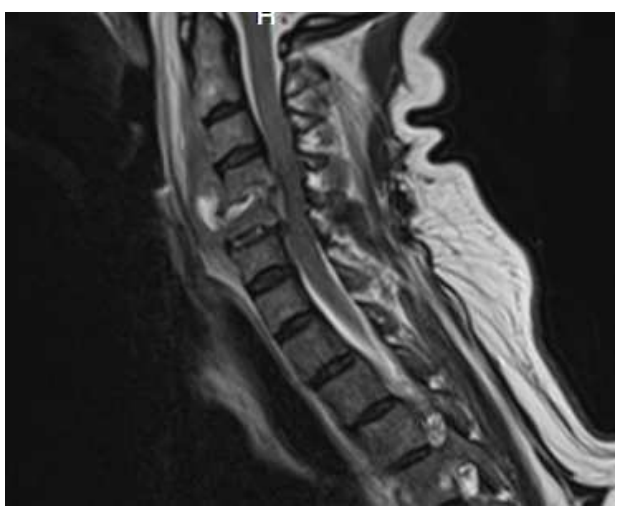

Figure-4: T2W Sag

Figure-3: Wedge compression of C4 and C5 vertebrae with destruction of the intervening discepidural collection is seen is seen.

Figure-4: Hyperintense prevertebral 


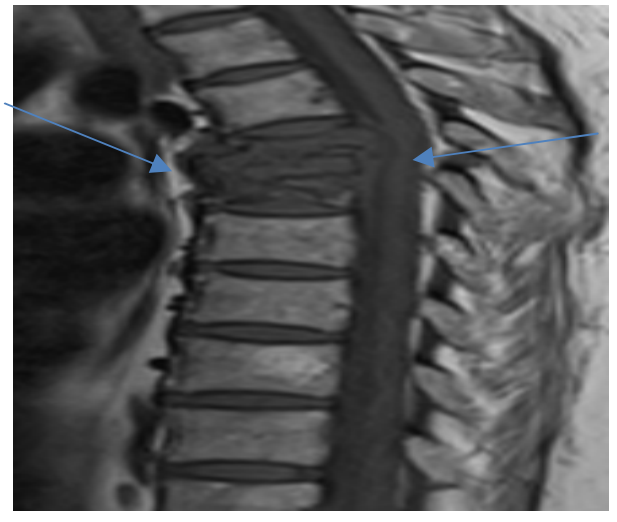

Figure-5: T1W Sag
Original Research Article

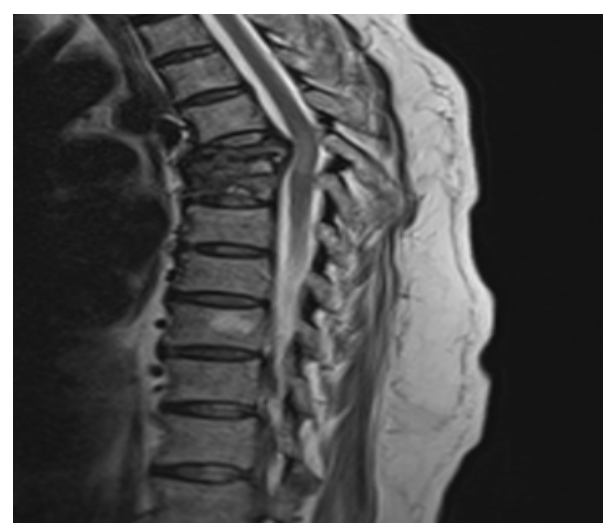

Figure-6: T2W Sag

Figure-5: Compression / Collapse of D5 and D6 vertebrae and hypointense signal intensityof involved vertebrae. Hypointense prevertebral and epidural collection is seen (Blue Arrow)

Figure-6: Epidural soft tissue is seencausing compression of spinalcausing compression of spinalcord.

\section{Discussion}

Tuberculosis is a major health issue being more common in developing countries and in the poor socioeconomic strata. Spinal TB constitutes the most common site of skeletal TB and presents with various neurological symptoms.

The present study was conducted on 25 patients in department of Radiodiagnosis, GMC and Rajindra hospital Patiala who were referred with history of fever, backache and features of neurological compression like bladder bowel involvement, limb weakness and paralysiswith varying degree of kyphotic and gibbus deformity. The aim was to evaluate the role of MRI in diagnosis and to study the spectrum of various findings that help in assessing the extent and severity of spinal involvement for planning early and accurate management.

Age of patients in the present study varied from 12 to 70 years with the mean age of 43.36 years. Males were more commonly affected with this disease. Out of 25 cases, $15(60 \%)$ were males and 10(40\%) were females with the mean age of 43.64 in males and 43 years in females. Toloba et al[6](2001) also reported in their study on 178 cases of spinal TB, the average age of patients as $41 \pm 15$ and a male predominance (102 men against 76 women) with a sex ratio of 1.3.

In the present study, the most common site of involvement was thoracic spine and was seen in $13(52 \%)$ out of 25 cases followed by lumbar (28\%), cervical spine $(16 \%)$ and we had one case of TB involving the sacral vertebrae as shown in table 1 . Rauf et al[7] (2015) also reported that the most common site of involvement of spinal TB was dorsal spine (45\%), followed by lumbosacral spine $(33 \%)$, cervical spine $(10 \%)$ and multiple level involvement seen in $12 \%$. The lesions were hypo to isointense on T1WI as shown in Figures 1,3 and 5and iso to hyperintense on T2 (figures2, 4and 6). Contrast enhanced MRI was very helpful in diagnosing paraspinal abscesses, granulation tissue and determining the level of vertebrae involved along with its signal intensities. The lesions show marked heterogenous or peripheral enhancement. In present study of 25 cases, vertebral body edema was seen in all the cases, disc involvement in the form of reduced disc height (as shown in figures 3 and 4) and enhancement on contrast scan was seenin $23(92 \%)$ cases. Similar pattern of involvement was seen by Rivas et al [8](2013) and Smith AS et al[9](1989) in their study on patients of spinal TB.

The multiplanar imaging capability of MR imaging greatly improves the detection of vertebral intraosseous abscesses, skip lesions, subligamentous spread of infection, and epidural extension commonly associated with tuberculous spondylitis[10].

Pre and paravertebral collection were seen in 22 patients which means in $88 \%$ of cases. Anterior subligamentous involvement was seen in 8 cases out of 25 patients. Paraplegia and sometimes quadriplegia were serious complications of the tuberculous spine seen in approximately $10 \%$ of patients. Copious epidural pus and granulation tissue alone or in combination with vertebral collapse, subluxation, or dislocationproduce cord compression. Rarely, the pus penetrates the dura resulting in severe meningomyelitis. 


\section{Original Research Article}

Reduced vertebral height with compression/collapse was in $64 \%$ of the cases. Epidural soft tissue formation was seen in $14(56 \%)$ out of 25 cases. Cord compression due to epidural granulation tissue or vertebral compression/collapse was seen in $11(44 \%)$ cases in the present study.

Similar studies on MRI features in spinal TB have been done by J Pursey et al[11](2010), Zaidi H et al[12] (2010) and Andronikou S et al[13](2002), and our study is consistent with findings of these observers.

Posterior element involvement was seen in 10 cases i.e. $40 \%$ of cases which is not uncommon and is associated with severe vertebral destruction and kyphotic deformity. Similar observations were alsoreported by Yusof MI et al[14](2009) andEhara S et al[15](1997).

It is important to differentiate tuberculous spondylitis from pyogenic spondylitis. MRI has been shown to be accurate in differentiating tuberculous spondylitis from pyogenic spondylitis.

The presence of a well-defined paraspinal abnormal signal, a thin and smooth abscess wall, subligamentous spread to three or more vertebral levels, and multiple vertebral or entire body involvement are more suggestive of tuberculous spondylitis than pyogenic spondylitis [16].

The MRI diagnosis was correlated with the other investigations for TB and with histopathological diagnosis done in few cases.

MRI is extremely useful in evaluating the extent of involvement and response to therapy of isolated TB of posterior elements. The lack of ionizing radiation and the multiplanar capability of MR imaging make it advantageous for postoperative assessment of the spine and follow-up studies for monitoring the response to therapy.

\section{Conclusion}

We conclude that in spinal tuberculosis, the superior contrast resolution of MR imaging is useful for showing contiguous vertebral involvement, skip lesions and paraspinal collections and the extent of the epidural pus in patients presenting with neurological deficits. Familiarity with the spectrum of MR findings in tuberculous spondylitis, especially in high risk patient population, can prevent a delay in diagnosis and may limit the morbidity that can be caused by this aggressive but curable infectious disease.
The present study suggested that MRI is a definitive diagnostic modality for evaluating spinal TB patients and is an effective tool to assess the severity and extent of the disease with its various radiological features.

Funding: Nil, Conflict of interest: None.

Permission of IRB: Yes

\section{References}

1. Rasouli MR, Mirkoohi M, Vaccaro AR, Yarandi KK, Rahimi-Movaghar V. Spinal tuberculosis: diagnosis and management.Asian Spine J.2012Dec;6(4):294-308. doi: 10.4184/asj.2012.6.4.294. Epub2012Dec 14.

2. Moorthy S, Prabhu NK. Spectrum of MR imaging findings in spinal tuberculosis. AJR Am J Roentgenol. 2002 Oct;179(4):979-83.

3. Ansari, S., Rauniyar, R.K., Dhungel, K., Sah, P.L.,. Chaudhary, P., Ahmad, K., Amanullah, M.F., 2013. MR evaluation of spinal tuberculosis. Al Ameen J. Med. Sci.; 6(3):219-25.

4. Garg RK, Somvanshi DS. Spinal tuberculosis: a review.J Spinal Cord Med. 2011;34(5):440-54. doi: 10.1179/2045772311Y.0000000023.

5. Ansari S, Amanullah MF, Ahmad K, Rauniyar RK. Pott's Spine: Diagnostic Imaging Modalities and Technology Advancements. N Am J Med Sci.2013 Jul; 5 (7): 404-11. doi: 10.4103/1947-2714.115775.

6. Toloba Y, Diallo S, Maiga Y, Sissoko B, Ouattara K, Soumaré D, Sidibé S. [Spinal tuberculosis (Pott's disease): epidemiological, clinical, radiological and evolutionary aspects at the University Hospital of Point G]. Mali Med.2011;26(2):8-11.

7. Rauf F, Chaudhry UR, Atif M, urRahaman M. Spinal tuberculosis: Our experience and a review of imaging methods.Neuroradiol J. 2015 Oct;28(5):498-503. doi: $10.1177 / 1971400915609874$. Epub 2015 Oct 8.

8. Rivas-Garcia A, Sarria-Estrada S, Torrents-Odin C, Casas-Gomila L, Franquet E. Imaging findings of Pott's disease. European Spine Journal. 2013 Jun 1; 22(4): 567-78.

9. Smith AS, Weinstein MA, Mizushima A, Coughlin B, Hayden SP, Lakin MM, Lanzieri CF. MR imaging characteristics of tuberculous spondylitis vs vertebral osteomyelitis. American Journal of Roentgenology. 1989 Aug 1;153(2):399-405. 


\section{Original Research Article}

10. Shanley DJ. Tuberculosis of the spine: imaging features.AJR Am JRoentgenol.1995Mar;164(3):659-64.

11. Jacqueline P and Sharon S. Potts disease: Diagnosis with magnetic resonance imaging. Radiography, 2010; 16:84-88.

12. Zaidi H, Akram MH, Wala MS. Frequency and magnetic resonance imaging patterns of tuberculous spondylitis lesions in adults. J Coll Physicians Surg Pak. 2010 May 1;20(5):303-6.

13. Andronikou S, Jadwat S, Douis H. Patterns of disease on MRI in 53 children with tuberculous spondylitis and the role of gadolinium. Pediatr Radiol. 2002 Nov; 32(11):798-805. Epub2002 Aug 17.
14. Yusof MI, Hassan E, Rahmat N, Yunus R. Spinal Tuberculosis: The Association between Pedicle Involvement and Anterior Column Damage and Kyphotic Deformity. Spine, 2009; 34: 713717.

15. Ehara S, Shimamura T, Wada T. Single vertebral compression and involvement of the posterior elements in tuberculous spondylitis: observation on MR imaging. Radiat Med. 1997 May-Jun;15(3):143-7.

16. Jung NY, Jee WH, Ha KY, Park CK, Byun JY. Discrimination of tuberculous spondylitis from pyogenic spondylitis on MRI.AJR Am J Roentgenol. 2004 Jun;182(6):1405-10.

\section{How to cite this article?}

Bhatnagar S, Garg A, Kaur A, Kaur N, Mohi J. Spinal tuberculosis: imaging features on MRI. Int J Med Res Rev 2018;6 (02):65-70. doi:10.17511/ijmrr. 2018.i02.01. 